\title{
Persönlichkeits- und Datenschutz: die irreversible Anonymisierung als ethisches Dilemma
}

\author{
Die Richtlinien «Biobanken» der Schweizerischen Akademie der Medizinischen Wissenschaften
}

B. Elger

Ende Mai 2005 hat die SAMW die neuen Richtlinien: «Biobanken: Gewinnung, Aufbewahrung und Nutzung von menschlichem biologischem Material für Ausbildung und Forschung» zur Vernehmlassung verabschiedet. Bis zum Vorliegen der definitiven Fassung der Richtlinien werden in verschiedenen Beiträgen bestimmte Aspekte des Richtlinienentwurfes vertieft behandelt. Der Richtlinienentwurf ist unter www.samw.ch online abrufbar.

Korrespondenz:

PD Dr. Bernice Elger

CMU

Médecine légale

Avenue de Champel 9

$\mathrm{CH}-1211$ Genève 4

E-Mail: bernice.elger@hcuge.ch
Persönlichkeits- und Datenschutz ist nicht nur wichtig, weil es ein Recht derjenigen ist, deren Gewebeproben und Daten $\mathrm{zu}$ Forschungszwecken genutzt werden. Es liegt auch im Interesse der Betreiber von Biobanken und Forschern selbst, das Vertrauen derjenigen aufrechtzuerhalten, ohne deren altruistische Zustimmung zur Nutzung ihrer Proben und Daten Forschung gar nicht stattfinden könnte.

Die SAMW hat sich bemüht, in ihren Richtlinien den erforderlichen Persönlichkeits- und Datenschutz in angemessener Form abzusichern, ohne dabei unüberwindliche Hürden für die Forschung entstehen zu lassen. Wie sich auch in der Arbeit anderer Kommissionen in Europa und auf internationaler Ebene gezeigt hat, ist dies kein leichtes Unterfangen.

Verschiedene Aspekte müssen berücksichtigt werden. Der Datenschutz wird in der Schweiz gesetzlich geregelt. Das Bundesgesetz über den Datenschutz vom 19. Juni 1992 (DSG) steht im Einklang mit allgemeinen europäischen Standards (Europäische Richtlinie 95/46/EC). Ausgenommen von Datenschutzbestimmungen sind Daten, die soweit anonymisiert sind, dass die Identität der Person, von der die Daten stammen, nicht mehr feststellbar ist. Die Anonymisierung von medizinischen Daten, vor allem aber auch die gesetzlich weniger klar geregelte Anonymisierung von Proben, sind jedoch z.Z. weit davon entfernt, eine Routineaufgabe zu sein [1]. Wie sich im Rahmen der isländischen Biobank gezeigt hat, ist es möglich - je nach der Häufigkeit bestimmter Merkmale in einer Population -, schon bei der Kombination weniger Eigenschaf- ten, z. B. Beruf, Tumorart und Wohnort, in einer Datenbank einzelne Personen zweifelsfrei zu identifizieren. Hinzu kommt das Problem, dass auch bei einer ausreichenden Anonymisierung des Individuums die Anonymität von Gruppen nicht unbedingt gewährleistet ist.

In Anbetracht der Wichtigkeit der zu schützenden Persönlichkeitsrechte empfehlen die Richtlinien der SAMW, im Falle von Biobanken durch eine zuständige Instanz [2] prüfen zu lassen, ob genügend Daten abgetrennt wurden, um die Anonymisierung als ausreichend zu bezeichnen. Für eine solche Beurteilung ist Expertenwissen erforderlich.

\section{Irreversible oder reversible Anonymisierung: die Debatte}

Die Ansichten darüber, wann eine irreversible Anonymisierung von Gewebeproben und Daten in der Forschung sinnvoll, ethisch wünschenswert oder aber auch bedenklich ist, gehen z.Z. weit auseinander. Biobankenexperten warnen in der Mehrheit vor irreversiblen Schritten. Denn wiederholt hat sich gezeigt, wie wichtig Probenund Datensammlungen für die Wissenschaft sind. Es war oft nicht absehbar, zu welchen wertvollen Zwecken diese Biobanken später in der Forschung dienen konnten.

Hinzu kommt, dass die irreversible Anonymisierung nicht allen Bedenken Rechnung trägt. Persönlichkeits- und Datenschutz soll nicht nur dem Schutz vor nachteiligen Konsequenzen dienen, die entstehen, wenn Daten in falsche Hände gelangen. Geschützt werden soll auch das Interesse von Spendern, mitzubestimmen, wofür eigenes Gewebe genutzt wird. Diskussionen und Gesetzesinitiativen in mehreren Ländern [3] zeigen, dass es als Eingriff in die Persönlichkeitsrechte erlebt wird, wenn eigenes Gewebe, selbst wenn dies irreversibel anonymisiert ist, für Zwecke verwendet wird, die vom betreffenden 
Spender als ethisch nicht akzeptabel angesehen werden, sei es im Fall von Studien zu genetischen Ursachen von Homosexualität, Aggressivität oder Alkoholismus oder auch bei jeglicher Forschung, die mit Klonen in Verbindung gebracht wird. Auf seiten derer, die ihre Proben und Daten der Wissenschaft zur Verfügung stellen, wird daher angeführt, dass ein Widerruf der Einwilligung für solche ethisch nicht von allen akzeptierten, aber später vielleicht geplanten Forschungsprojekten nur möglich ist, wenn Proben nicht irreversibel anonymisiert sind.

Als Argument gegen die irreversible Anonymisierung wird ausserdem genannt, dass dieser Prozess es unmöglich macht, Gewebespender später zu benachrichtigen, sollten individuell für sie medizinisch bedeutsame Forschungsergebnisse anfallen. Es wird ebenfalls angeführt, dass im Falle von DNA-Proben selbst irreversible Anonymisierung keinen vollständigen Schutz vor Identifizierung bietet (DNA-Fingerprinting), sondern nur einen relativen Schutz, der nicht signifikant über den Schutz durch eine reversible sichere Kodierung hinausgeht.

\section{Irreversible oder reversible \\ Anonymisierung: die Rolle der Überprüfungsinstanz}

Die genannten Argumente erschienen der SAMW schwerwiegend genug, um grundsätzlich Mechanismen vorzuschlagen, die helfen sollen, die in der Vergangenheit als nachteilig erlebten Vorgehensweisen in der Zukunft zu vermeiden und damit sowohl der Forschung als auch den Spendern von Proben und Daten von Nutzen zu sein. Die Richtlinien schlagen daher vor, dass die Überprüfungsinstanz [2] die Verwendung von irreversibel anonymisierten (und auch von nichtkodierten) Proben und Daten genehmigt und der Spender ihr zustimmt.

Sicher gibt es viele Forschungsprojekte, bei denen eine irreversible Anonymisierung eine ethisch akzeptable Lösung ist und unnötige Kosten vermeiden lässt. Dies ist im allgemeinen der Fall bei der Nutzung von Proben zur Evaluation von neuen Messmethoden. Die irreversible Anonymisierung soll auf keinen Fall generell ausgeschlossen werden, sondern soll weiter eine Option für viele Forschungsprojekte bleiben. Dies sollten jedoch nicht die Forscher selbst festlegen. Die SAMW erachtet es als unabdingbar, ein deutliches Signal zu setzen, dass eine irreversible Anonymisierung niemals banal ist, weder aus wissenschaftlicher, noch aus juristischer oder ethischer Sicht. Für jedes Forschungsprojekt sollte individuell die Indikation zu einer solchen Anonymisierung erstellt werden, nicht zuletzt, um zu vermeiden, dass Forscher den Weg der irreversiblen Anonymisierung nur aus dem Grund wählen, um bei schon bestehenden Banken und Gewebeproben, die für andere als die ursprünglich geplanten Zwecke verwendet werden, die in Kapitel 10 der Richtlinien geforderte generelle Bewilligung nicht einholen zu müssen.

\section{Reversible Anonymisierung (Kodierung) als Alternative zur irreversiblen Anonymisierung?}

Die Alternative zur irreversiblen Anonymisierung, die in den Richtlinien (Kapitel 4.2) empfohlen wird, ist die Kodierung (britische und europäische Dokumente sprechen hier auch von reversibler Anonymisierung [4]): «Daten und Proben sollen durch eine sichere Form der Kodierung wirksam geschützt werden [5]. Dies gilt sowohl für die Aufbewahrung in einer Biobank als auch bei der Nutzung in Ausbildung und Forschung. Der Kode ist von den Daten organisatorisch getrennt aufzubewahren und zu verwalten.»

«Sichere Form der Kodierung» impliziert, dass, wie bei der irreversiblen Anonymisierung, alle persönlichen Daten, die eine Identifizierung des Spenders ermöglichen, abgetrennt werden. Mittels eines Kodierungsschlüssels ist es jedoch möglich, wenn nötig die Verbindung zu den die Identifizierung ermöglichenden Daten wiederherzustellen. Um eine missbräuchliche Weitergabe des Kodierungsschlüssels an Unbefugte zu verhindern, sollte dieser Schlüssel «in der Hand eines deklarierten Geheimnisträgers sein», der von der sekundären Nutzung unabhängig ist, und damit nicht in Interessenkonflikte gerät.

\section{Klare Regelung für den Transfer von Proben und Daten}

Abgesehen von einer sicheren Kodierung werden international von Biobankenexperten weitere Massnahmen empfohlen, welche die SAMW ebenfalls in ihre Richtlinien aufgenommen hat. Zum Schutz der Spender vor unkontrollierter Verwendung von Proben und Daten aus Biobanken ist es wichtig, den Transfer von Proben und Daten an Dritte klar zu regeln. «Der Hauptverantwortliche für die Biobank (Leiter) oder das primäre Forschungsprojekt (Prüfer) überlässt Proben oder Daten anderen Forschern für sekundäre Projekte keinesfalls in nicht kodierter Form. 
Diese Forscher sind verpflichtet, Proben und Probenreste, die sie nicht verbraucht haben, der Biobank zurückzusenden oder sie zu vernichten. Es ist ihnen nicht gestattet, Proben oder Daten an Dritte weiterzugeben.» Werden Proben oder Daten ins Ausland weitergegeben, muss vorher sichergestellt sein, «dass die Standards gemäss den vorliegenden Richtlinien eingehalten werden».

Um die Sicherheitsmassnahmen kontrollieren zu können, gilt folgende Grundregel: «Jeder Transfer von Proben und Daten muss nachvollziehbar dokumentiert werden.» Diese Bestimmung ist ausserdem unabdingbar, um das Widerrufsrecht und die Wahrung der Persönlichkeitsrechte des Spenders zu gewährleisen. Der Geheimnisträger sollte «jederzeit nachvollziehen können, wo sich Proben und Daten befinden».

Einige Reaktionen auf die vorgeschlagenen Richtlinien zeigen, dass manche Forscher der Ansicht sind, dass ein ausreichender Persönlichkeits- und Datenschutz auch durch weniger umfangreiche Massnahmen wirkungsvoll erreicht werden kann. Hier kann nur festgestellt werden, dass die Schweizer Richtlinien nicht nur den weitgehend überschaubaren Strukturen innerhalb der Schweiz Rechnung tragen müssen, sondern auch der Tatsache, dass Biobanken und die damit verbundenen Forschungsprojekte ihren vollen Nutzen nur in einem internationalen Rahmen entfalten können. Eine weltweite Ga- rantie der Datensicherheit und des Respekts der Persönlichkeitsrechte von Gewebespendern wird sich für alle, auch Forscher und Biobankenverantwortliche, lohnen, weil nur auf diese Weise Vertrauen geschaffen werden kann, ein Widerruf der Zustimmung äusserst selten bleibt und internationale Projekte wirkungsvoll unterstützt werden können.

\section{Literatur}

1 Wilson P. Commentary: Legal issues of data anonymisation in research. BMJ 2004;328:1300-1.

2 Zurzeit übernehmen diese Aufgabe die kantonalen Ethikkommissionen für klinische Versuche gemäss Art. 57 HMG.

3 Trouet C. New European guidelines for the use of stored human biological materials in biomedical research. J Med Ethics 2004;30:99-103.

4 Council of Europe Steering Committee on Bioethics (CDBI), Evans I, Roscam Abbing $\mathrm{H}$. Proposal for an instrument on the use of archived human biological materials in biomedical research 2002: www.coe.int/T/E/Legal_affairs/Legal_ co-operation/Bioethics/Activities/Biomedical_ research/CDBI-INF(2002)5E.pdf; Medical Research Council (MRC). Ethics Series. Personal Information in Medical Research 2000: www.mrc.ac.uk/ pdf-pimr.pdf (accessed April 2005).

5 Eine leicht entzifferbare Kodierung wie beispielsweise Kodenummer, die sich aus Initialen und Geburtsdatum des Spenders zusammensetzt, stellt keinen ausreichenden Schutz dar. Siehe auch Committee for Proprietary Medicinal Products (CPMP). Position Paper on Terminology in Pharmacogenetics. 2002. www.emea.eu.int. 